IMA Journal of Numerical Analysis (2013) Page 1 of 19

doi:10.1093/imanum/drn000

\title{
Adaptive discontinuous Galerkin methods for non-stationary convection-diffusion problems
}

\author{
Andrea Cangiani, Emmanuil H. Georgoulis and Stephen Metcalfe \\ Department of Mathematics, University of Leicester, University Road, Leicester LE1 7RH, \\ United Kingdom
}

[Received on 24 September 2013]

\begin{abstract}
This work is concerned with the derivation of a robust a posteriori error estimator for a discontinuous Galerkin method discretisation of a linear non-stationary convection-diffusion initial/boundary value problem and with the implementation of a corresponding adaptive algorithm. More specifically, we derive a posteriori bounds for the error in the $L^{2}\left(H^{1}\right)+L^{\infty}\left(L^{2}\right)$-type norm for an interior penalty discontinuous Galerkin (dG) discretisation in space and a backward Euler discretisation in time. Finally, an adaptive algorithm is proposed utilising the error estimator. Optimal rate of convergence of the adaptive algorithm is observed in a number of test problems and for various Pèclet numbers.
\end{abstract}

Keywords: Discontinuous Galerkin; unsteady convection-diffusion; a posteriori error estimation; adaptive finite element methods.

\section{Introduction}

The interaction between convection and diffusion modelled by initial/boundary value problems involving partial differential equations (PDEs) poses a number of challenges in the context of their numerical approximation. Indeed, stationary convection-dominated convection-diffusion type problems admit analytical solutions of a multiscale nature that can contain steep gradients, usually termed boundary or interior layers, depending upon their location in the computational domain. The accurate and efficient numerical resolution of such steep layers is a challenge as their exact location cannot be, in general, known a priori. In the special cases where the location of boundary or interior layers is known, structured grids have been successfully employed (Roos et al. (2008)). Ultimately, in non-stationary convection-diffusion equations, the nature of the solution (including layers) may vary throughout the domain as time progresses. This renders the use of adaptive algorithms an attractive proposition for the accurate and efficient numerical approximation of this class of problems. As adaptive algorithms are usually based on suitable a posteriori error estimators, the formulation of estimators that can robustly estimate both the temporal and spatial nature of the error are of particular interest.

A posteriori error estimation for stationary linear equations is now relatively well understood; for pure diffusion problems there is a huge array of estimators available for a wide variety of different types of finite element discretisations (Verfürth (1996); Ainsworth \& Oden (2000)) and for dG methods in particular (Karakashian \& Pascal (2003); Becker et al. (2003); Houston et al. (2007)). For stationary convection-diffusion equations, the quest for robust a posteriori error estimators (in the sense that they are independent of the Péclet number of the problem) has seen recent advancements in various contexts (Verfürth (1998, 2005b); Kunert (2003); Sangalli (2008); Schötzau \& Zhu (2009); Ern et al. (2010); Schötzau \& Zhu (2011)).

A posteriori error estimators for non-stationary linear convection-diffusion equations are also avail- 
able for various discretisations (Houston \& Süli (2001); Berrone \& Canuto (2004); Verfürth (2005a); Ern \& Proft (2005); Araya et al. (2005a,b); Sun \& Wheeler (2006); Georgoulis et al. (2008); Lozinski et al. (2009)). A posteriori error estimators for (spatial) dG methods for non-stationary pure diffusion problems can be found in Georgoulis et al. (2011); Ern \& Vohralík (2010); Šebestová (2012), while convection-diffusion problems are considered in Dolejší et al. (2013).

This work is concerned with the derivation and implementation of an a posteriori estimator in the $L^{2}\left(H^{1}\right)+L^{\infty}\left(L^{2}\right)$-type norm for the backward Euler discretisation in time (zero-th order dG method) and the interior penalty $\mathrm{dG}$ discretisation in space of the non-stationary linear convection-diffusion equation with variable coefficients. The derivation of the a posteriori bound utilises the elliptic reconstruction technique (Makridakis \& Nochetto (2003); Georgoulis et al. (2011)) which allows the use of the robust elliptic error estimator from Schötzau \& Zhu (2009). The temporal residual is also treated so as to ensure that robustness, in the sense that the time-effectivity indices are insensitive to the Péclet number of the problem, is maintained - this is highlighted numerically. The resulting residual-based a posteriori error estimator is shown numerically to be reliable and efficient in both time and space. The a posteriori estimator derived below can be viewed as the analogue of the one presented in Verfürth (2005a) when the spatial discretisation is a dG method. The a posteriori estimator in Dolejší et al. (2013) is based on the flux reconstruction framework for a stronger norm.

The a posteriori estimator is used to drive an adaptive algorithm which is numerically assessed in two ways. First, the savings with respect to the degrees of freedom and time steps by using space-time adaptivity are shown in a series of test problems. Second, the rates of convergence of the space-time adaptive algorithm are computed. The adaptive algorithm appears to converge optimally in both space and time. To the best of our knowledge, numerically verified optimal convergence rates for practical adaptive algorithms for $\mathrm{dG}$ methods for parabolic problems have not appeared before in the literature.

The remainder of this work is structured as follows. The function space setting and the model problem are given in Section 2. Section 3 describes the discretisation of the problem. In Section 4 we state an a posteriori error bound for the stationary elliptic problem. Sections 5 and 6 contain the derivation of the a posteriori bounds for the non-stationary convection-diffusion model problem in the semi-discrete and the fully-discrete settings, respectively. Section 7 describes a space-time adaptive algorithm driven by the a posteriori estimators derived in the previous section. A series of numerical experiments are presented in Section 8 and the final conclusions are drawn in Section 9.

\section{Model problem}

Let the computational domain $\Omega \subset \mathbb{R}^{2}$ be a bounded Lipschitz polygon with boundary $\partial \Omega$.

We denote the standard $L^{2}$-inner product on $\Omega$ by $(\cdot, \cdot)$ and the standard $L^{2}$-norm on $\Omega$ by $\|\cdot\|$. For $1 \leqslant p \leqslant+\infty$, we define the spaces $L^{p}(0, T ; X)$ (where $X$ is a real Banach space with norm $\|\cdot\|_{X}$ ) that consist of all measurable functions $v:[0, T] \rightarrow X$ for which:

$$
\begin{aligned}
& \|v\|_{L^{p}(0, T ; X)}:=\left(\int_{0}^{T}\|v(t)\|_{X}^{p} d t\right)^{1 / p}<+\infty, \quad \text { for } \quad 1 \leqslant p<+\infty \\
& \|v\|_{L^{\infty}(0, T ; X)}:=\underset{0 \leqslant t \leqslant T}{\operatorname{ess} \sup }\|v(t)\|_{X}<+\infty, \quad \text { for } \quad p=+\infty
\end{aligned}
$$

We also define $H^{1}(0, T, X):=\left\{u \in L^{2}(0, T ; X): u_{t} \in L^{2}(0, T ; X)\right\}$. Finally, we denote by $C(0, T ; X)$ and $C^{0,1}(0, T ; X)$, respectively, the spaces of continuous and Lipschitz-continuous functions $v:[0, T] \rightarrow X$ 
such that:

$$
\begin{gathered}
\|v\|_{C(0, T ; X)}=\max _{0 \leqslant t \leqslant T}\|v(t)\|_{X}<\infty, \\
\|v\|_{C^{0,1}(0, T ; X)}=\max \left\{\|v\|_{C(0, T ; X)},\left\|\frac{\partial v}{\partial t}\right\|_{C(0, T, X)}\right\}<\infty .
\end{gathered}
$$

We consider the model problem of finding $u: \Omega \rightarrow \mathbb{R}$ such that:

$$
\begin{aligned}
\frac{\partial u}{\partial t}-\varepsilon \Delta u+\mathbf{a} \cdot \nabla u+b u & =f & & \text { in } \Omega \times(0, T], \\
u & =0 & & \text { on } \partial \Omega \times(0, T], \\
u(\cdot, 0) & =u_{0} & & \text { in } \bar{\Omega} .
\end{aligned}
$$

We make the following assumptions: $0<\varepsilon \leqslant 1, f \in C\left(0, T ; L^{2}(\Omega)\right), \mathbf{a} \in C\left(0, T ; W^{1, \infty}(\Omega)\right)^{2}, b \in$ $C\left(0, T ; L^{\infty}(\Omega)\right)$ and $u_{0} \in L^{2}(\Omega)$.

It is assumed that the length of $\mathbf{a} \equiv \mathbf{a}(\mathbf{x}, \mathbf{t})$ and the area of $\Omega$ are of order one, possibly up to rescaling, so that $\varepsilon^{-1}$ can be taken to be the Péclet number of the problem. For simplicity, we assume that there are constants $\beta \geqslant 0$ and $c_{*} \geqslant 0$ such that:

$$
b-\frac{1}{2} \nabla \cdot \mathbf{a} \geqslant \beta \quad \text { a.e. in } \Omega \times[0, T], \quad\|-\nabla \cdot \mathbf{a}+b\|_{C\left(0, T ; L^{\infty}(\Omega)\right)} \leqslant c_{*} \beta .
$$

The weak form of (2.3) then reads: find $u \in L^{2}\left(0, T ; H_{0}^{1}(\Omega)\right) \cap H^{1}\left(0, T ; L^{2}(\Omega)\right)$ such that for each $t \in(0, T]$ we have

$$
\int_{\Omega} \frac{\partial u}{\partial t} v d x+\int_{\Omega}(\varepsilon \nabla u \cdot \nabla v+\mathbf{a} \cdot \nabla u v+b u v) d x=\int_{\Omega} f v d x \quad \forall v \in H_{0}^{1}(\Omega) .
$$

Under the regularity assumptions above, we have that $u \in C\left(0, T ; L^{2}(\Omega)\right)$.

\section{Discontinuous Galerkin method}

Let the mesh $\zeta=\{K\}$ be a shape-regular subdivision of $\Omega$, with $K$ denoting a generic element. We assume that the subdivision $\zeta$ is constructed via affine mappings $F_{K}: \hat{K} \rightarrow K$ with non-singular Jacobian where $\hat{K}$ is the reference triangle or the reference square. The mesh is allowed to contain a uniformly fixed number of regular hanging nodes per edge. We define the finite element space

$$
V_{h} \equiv V_{h}(\zeta):=\left\{v \in L^{2}(\Omega):\left.v\right|_{K} \circ F_{K} \in \mathscr{P}^{p}(\hat{K}), K \in \zeta\right\},
$$

where $\mathscr{P}^{p}(K)$ is the space of polynomials of total degree $p$ if $\hat{K}$ is the reference triangle, or the space of polynomials of degree $p$ in each variable if $\hat{K}$ is the reference square.

Denote by $\mathscr{E}(\zeta)$ the set of all edges in the triangulation $\zeta$ and $\mathscr{E}$ int $(\zeta) \subset \mathscr{E}(\zeta)$ the subset of all interior edges. We also denote the diameter of an element $K$ by $h_{K}$ and the length of an edge $E$ by $h_{E}$. The outward unit normal on the boundary $\partial K$ of an element $K$ is denoted by $\mathbf{n}_{K}$. Given an edge $E \in$ $\mathscr{E}^{\operatorname{eint}}(\zeta)$ shared by two elements $K$ and $K^{\prime}$, a vector field $\mathbf{v} \in\left[H^{1 / 2}(\Omega)\right]^{2}$ and a scalar field $v \in H^{1 / 2}(\Omega)$, we define jumps and averages of $\mathbf{v}$ and $v$ across $E$ by:

$$
\begin{array}{rlrl}
\{\mathbf{v}\} & =\frac{1}{2}\left(\left.\mathbf{v}\right|_{\bar{K}}+\left.\mathbf{v}\right|_{\bar{K}^{\prime}}\right), & & {[\mathbf{v}]=\left.\mathbf{v}\right|_{\bar{K}} \cdot \mathbf{n}_{K}+\left.\mathbf{v}\right|_{\bar{K}^{\prime}} \cdot \mathbf{n}_{K^{\prime}},} \\
\{v\}=\frac{1}{2}\left(\left.v\right|_{\bar{K}}+\left.v\right|_{\bar{K}^{\prime}}\right), & {[v]=\left.v\right|_{\bar{K}} \mathbf{n}_{K}+\left.v\right|_{\bar{K}^{\prime}} \mathbf{n}_{K^{\prime}} .}
\end{array}
$$


4 of 19

If $E \subset \partial \Omega$, we set $\{\mathbf{v}\}=\mathbf{v},[\mathbf{v}]=\mathbf{v} \cdot \mathbf{n},\{v\}=v$ and $[v]=v \mathbf{n}$, with $\mathbf{n}$ denoting the outward unit normal to the boundary $\partial \Omega$.

We define the inflow and outflow parts of the boundary $\partial \Omega$ at the time $t$, respectively, by:

$$
\partial \Omega_{\text {in }}^{t}=\{x \in \partial \Omega \mid \mathbf{a}(x, t) \cdot \mathbf{n}(x)<0\}, \quad \partial \Omega_{\text {out }}^{t}=\{x \in \partial \Omega \mid \mathbf{a}(x, t) \cdot \mathbf{n}(x) \geqslant 0\} .
$$

The inflow and outflow parts of an element $K$ at time $t$ are similarly defined as:

$$
\partial K_{\text {in }}^{t}=\left\{x \in \partial K \mid \mathbf{a}(x, t) \cdot \mathbf{n}_{K}(x)<0\right\}, \quad \partial K_{\text {out }}^{t}=\left\{x \in \partial K \mid \mathbf{a}(x, t) \cdot \mathbf{n}_{K}(x) \geqslant 0\right\} .
$$

The semi-discrete discontinuous Galerkin approximation to (2.5) then reads as follows. For $t=0$, set $u_{h}(0) \in V_{h}$ to be a projection of $u_{0}$ onto $V_{h}$. For $t \in(0, T]$, find $u_{h} \in C^{0,1}\left(0, T ; V_{h}\right)$ such that

$$
\left(\frac{\partial u_{h}}{\partial t}, v_{h}\right)+B\left(t ; u_{h}, v_{h}\right)+K_{h}\left(u_{h}, v_{h}\right)=\left(f, v_{h}\right) \quad \forall v_{h} \in V_{h},
$$

with

$$
\begin{aligned}
B(t ; w, v):= & \sum_{K \in \zeta} \int_{K}(\varepsilon \nabla w-\mathbf{a} w) \cdot \nabla v+(b-\nabla \cdot \mathbf{a}) w v d x+\sum_{E \in \mathscr{E}(\zeta)} \frac{\gamma \varepsilon}{h_{E}} \int_{E}[w] \cdot[v] d s \\
& +\sum_{K \in \zeta}\left(\int_{\partial K_{\text {out }}^{t} \cap \partial \Omega_{\text {out }}^{t}} \mathbf{a} \cdot \mathbf{n}_{K} w v d s+\int_{\partial K_{\text {out }}^{t} \backslash \partial \Omega} \mathbf{a} \cdot \mathbf{n}_{K} w\left(\left.v\right|_{\bar{K}}-\left.v\right|_{\bar{K}^{\prime}}\right) d s\right), \\
K_{h}(w, v):= & -\sum_{E \in \mathscr{E}(\zeta)} \int_{E}\{\varepsilon \nabla w\} \cdot[v]+\{\varepsilon \nabla v\} \cdot[w] d s,
\end{aligned}
$$

where $\bar{K} \cap \bar{K}^{\prime}=E \subset \partial K_{\text {out }}^{t} \backslash \partial \Omega$. In standard fashion, the penalty parameter $\gamma>0$ is chosen large enough so that the operator $B+K_{h}$ is coercive. Moreover, for simplicity of the presentation, we assume $\gamma>1$ so that the constants in the subsequent discussion are independent of it.

We note that the bilinear form $K_{h}$ is not well-defined for arguments in $H_{0}^{1}(\Omega)$, but the bilinear form $B$ is and for $u, v \in H_{0}^{1}(\Omega)$ and $t \in(0, T]$, we have

$$
B(t ; u, v)=\int_{\Omega}(\varepsilon \nabla u \cdot \nabla v+\mathbf{a} \cdot \nabla u v+b u v) d x .
$$

In light of this, the weak problem (2.5) can be rewritten for each $t \in(0, T]$ as

$$
\left(\frac{\partial u}{\partial t}, v\right)+B(t ; u, v)=(f, v) \quad \forall v \in H_{0}^{1}(\Omega) .
$$

We shall also consider a full discretisation of problem (2.5) by using a backward Euler method to approximate the time derivative.

To this end, consider a subdivision of $[0, T]$ into time intervals of lengths $\tau_{1}, \tau_{2}, \ldots, \tau_{n}$ such that $\sum_{j=1}^{n} \tau_{j}=T$ for some $n \geqslant 1$ and set $t^{0}=0$ and $t^{k}:=\sum_{j=1}^{k} \tau_{k}$. Denote an initial triangulation by $\zeta^{0}$. We associate to each time step $k>0$ a triangulation $\zeta^{k}$ which is assumed to have been obtained from $\zeta^{k-1}$ by locally refining and coarsening $\zeta^{k-1}$. This restriction upon mesh change is made to avoid degradation of the finite element solution, $c f$. Dupont (1982). To each mesh $\zeta^{k}$, we assign the finite element space $V_{h}^{k}=V_{h}\left(\zeta^{k}\right)$ given by (3.1). We also set $f\left(., t^{k}\right)=f^{k}, \mathbf{a}\left(., t^{k}\right)=\mathbf{a}^{k}$, and $b\left(., t^{k}\right)=b^{k}$ for brevity.

The fully-discrete dG method then reads as follows. Set $u_{h}^{0}$ to be a projection of $u_{0}$ onto $V_{h}^{0}$. For $k=0, \ldots, n-1$, find $u_{h}^{k+1} \in V_{h}^{k+1}$ such that

$$
\left(\frac{u_{h}^{k+1}-u_{h}^{k}}{\tau_{k+1}}, v_{h}^{k+1}\right)+B\left(t^{k+1} ; u_{h}^{k+1}, v_{h}^{k+1}\right)+K_{h}\left(u_{h}^{k+1}, v_{h}^{k+1}\right)=\left(f^{k+1}, v_{h}^{k+1}\right) \quad \forall v_{h}^{k+1} \in V_{h}^{k+1} .
$$


We shall take $u_{h}^{0}$ to be the orthogonal $L^{2}$-projection of $u_{0}$ onto $V_{h}^{0}$, although other projections onto $V_{h}^{0}$ can also be used.

\section{Error bounds for the dG method for the stationary problem}

To analyse the spatial error, we introduce the following quantities:

$$
\begin{aligned}
\|u\| \|: & :=\left(\sum_{K \in \zeta}\left(\varepsilon\|\nabla u\|_{L^{2}(K)}^{2}+\beta\|u\|_{L^{2}(K)}^{2}\right)+\sum_{E \in \mathscr{E}(\zeta)} \frac{\gamma \varepsilon}{h_{E}}\|[u]\|_{L^{2}(E)}^{2}\right)^{1 / 2}, \\
|u|_{A} & :=\left(\left(\sup _{v \in H_{0}^{1}(\Omega) \backslash\{0\}} \frac{\int_{\Omega} \mathbf{a} u \cdot \nabla v d x}{\|\| v \| \mid}\right)^{2}+\sum_{E \in \mathscr{E}(\zeta)}\left(\beta h_{E}+\frac{h_{E}}{\varepsilon}\right)\|[u]\|_{L^{2}(E)}^{2}\right)^{1 / 2} .
\end{aligned}
$$

We note that ||$|\cdot|||$ and $|\cdot|_{A}$ define norms on $H_{0}^{1}(\Omega)+V_{h}$.

Throughout this work, the symbols $\lesssim$ and $\gtrsim$ are used to denote inequalities true up to a positive constant independent of $\varepsilon, \beta, u$, and $u_{h}$.

Let $t \in(0, T]$ be fixed. It is easy to see that the bilinear form $B(t ; \cdot, \cdot)$ is coercive on $H_{0}^{1}(\Omega)$, viz.,

$$
B(t ; v, v) \geqslant\|\| v \|\left.\right|^{2},
$$

for all $v \in H_{0}^{1}(\Omega)$, and is continuous in the following sense:

$$
B(t ; w, v) \lesssim\left(|||w|||+|w|_{A}\right)|||v|||,
$$

for all $w \in H_{0}^{1}(\Omega)+V_{h}$ and $v \in H_{0}^{1}(\Omega)$. Moreover, the discrete bilinear form is coercive for $v_{h}$ in $V_{h}$ with respect to the $\||\cdot|\|$ norm, viz.,

$$
B\left(t ; v_{h}, v_{h}\right)+K_{h}\left(v_{h}, v_{h}\right) \gtrsim\left\|\left|v_{h}\right|\right\|^{2}
$$

Next, we introduce the following notation which will be used to define the a posteriori estimators:

$$
\alpha_{K}:=\min \left(h_{K} \varepsilon^{-\frac{1}{2}}, \beta^{-\frac{1}{2}}\right), \quad \alpha_{E}:=\min \left(h_{E} \varepsilon^{-\frac{1}{2}}, \beta^{-\frac{1}{2}}\right) \quad \text { and } \quad \alpha_{T}=\min \left(\varepsilon^{-\frac{1}{2}}, \beta^{-\frac{1}{2}}\right) .
$$

An a posteriori estimator for the stationary problem inspired by Schötzau \& Zhu (2009) will be utilised in our analysis. More specifically, we have the following result whose proof is completely analogous to the one of Theorem 3.2 in Schötzau \& Zhu (2009) and is therefore omitted for brevity.

THEOREM 4.1 For a given $t \in(0, T]$, let $u^{s} \in H_{0}^{1}(\Omega)$ be such that

$$
B\left(t ; u^{s}, v\right)=(f, v) \quad \forall v \in H_{0}^{1}(\Omega),
$$

and consider $u_{h}^{s} \in V_{h}$ such that

$$
B\left(t ; u_{h}^{s}, v_{h}\right)+K_{h}\left(u_{h}^{s}, v_{h}\right)=\left(f, v_{h}\right) \quad \forall v_{h} \in V_{h} .
$$

Then the following a posteriori bound holds:

$$
\begin{aligned}
\left(\left|\left\|u^{s}-u_{h}^{s}|\||+\left|u^{s}-u_{h}^{s}\right|_{A}\right)^{2} \lesssim\right.\right. & \sum_{K \in \zeta} \alpha_{K}^{2}\left\|f+\varepsilon \Delta u_{h}^{s}-\mathbf{a} \cdot \nabla u_{h}^{s}-b u_{h}^{s}\right\|_{L^{2}(K)}^{2}+\sum_{E \in \mathscr{E} \text { int }(\zeta)} \varepsilon^{\frac{3}{2}} \alpha_{E} \|\left.\left[\nabla u_{h}^{s}\right]\right|_{L^{2}(E)} ^{2} \\
& +\sum_{E \in \mathscr{E}(\zeta)}\left(\frac{\gamma \varepsilon}{h_{E}}+\beta h_{E}+\frac{h_{E}}{\varepsilon}\right)\left\|\left[u_{h}^{s}\right]\right\|_{L^{2}(E)}^{2} .
\end{aligned}
$$


6 of 19

\section{An a posteriori bound for the semi-discrete method}

To highlight the main ideas, we begin by deriving an a posteriori bound for the semi-discrete problem. The error will be analysed in the $L^{2}\left(H^{1}\right)+L^{\infty}\left(L^{2}\right)$ type norm

$$
\|u\|_{*}=\left(\|u\|_{L^{\infty}\left(0, T ; L^{2}(\Omega)\right)}^{2}+\int_{0}^{T} \mid\|u\| \|^{2} d t\right)^{1 / 2}
$$

Definition 5.1 For each $t \in(0, T]$, we define the elliptic reconstruction $w \in H_{0}^{1}(\Omega)$ to be the (unique) solution of the problem

$$
B(t ; w, v)=\left(f-\frac{\partial u_{h}}{\partial t}, v\right) \quad \forall v \in H_{0}^{1}(\Omega) .
$$

REMARK 5.1 The dG discretisation of the above equation is to find a function $w_{h} \in C^{0,1}\left(0, T, V_{h}\right)$ such that for each $t \in(0, T]$ we have

$$
B\left(t ; w_{h}, v_{h}\right)+K_{h}\left(w_{h}, v_{h}\right)=\left(f-\frac{\partial u_{h}}{\partial t}, v_{h}\right) \quad \forall v_{h} \in V_{h} .
$$

This, in conjunction with (3.5) and (4.4), implies that $w_{h}=u_{h}$. Thus, ||$\left|w-u_{h}\right|||+\left|w-u_{h}\right|_{A}$ can be estimated using Theorem 4.1.

We decompose the error as follows:

$$
e=u-u_{h}=\rho+\theta, \text { with } \rho:=u-w \text { and } \theta:=w-u_{h} .
$$

The dG solution, $u_{h}$, admits a decomposition into a conforming part $u_{h, c} \in H_{0}^{1}(\Omega) \cap V_{h}$ and a nonconforming part $u_{h, d} \in V_{h}$ with $u_{h}=u_{h, c}+u_{h, d}$, such that:

$$
\begin{aligned}
\left\|\left.\left|u_{h, d}\|\|^{2}+\right| u_{h, d}\right|_{A} ^{2}\right. & \lesssim \sum_{E \in \mathscr{E}(\zeta)}\left(\frac{\gamma \varepsilon}{h_{E}}+\beta h_{E}+\frac{h_{E}}{\varepsilon}\right)\left\|\left[u_{h}\right]\right\|_{L^{2}(E)}^{2}, \\
\left\|\frac{\partial u_{h, d}}{\partial t}\right\|^{2} & \lesssim \sum_{E \in \mathscr{E}(\zeta)} h_{E}\left\|\left[\frac{\partial u_{h}}{\partial t}\right]\right\|_{L^{2}(E)}^{2}, \\
\left\|u_{h, d}\right\|^{2} & \lesssim \sum_{E \in \mathscr{E}(\zeta)} h_{E}\left\|\left[u_{h}\right]\right\|_{L^{2}(E)}^{2} .
\end{aligned}
$$

We refer to Schötzau \& Zhu (2009) for proof of these estimates which are based on respective constructions by Karakashian \& Pascal (2003) and Verfürth (2005b). We further define $e_{c}:=u-u_{h, c}$ and $\theta_{c}:=w-u_{h, c}$.

LEMmA 5.1 For each $t \in(0, T]$ and for all $v \in H_{0}^{1}(\Omega)$ we have

$$
\left(\frac{\partial e}{\partial t}, v\right)+B(t ; \rho, v)=0 .
$$

Proof. This follows directly from Definition 5.1 and (3.8).

We define the error estimator, $\tilde{\eta}$, by

$$
\tilde{\eta}^{2}=\|e(0)\|^{2}+\int_{0}^{T} \tilde{\eta}_{S_{1}}^{2} d t+\min \left\{\left(\int_{0}^{T} \tilde{\eta}_{S_{2}} d t\right)^{2}, \alpha_{T}^{2} \int_{0}^{T} \tilde{\eta}_{S_{2}}^{2} d t\right\}+\max _{0 \leqslant t \leqslant T} \tilde{\eta}_{S_{3}}^{2},
$$


where

$$
\begin{aligned}
\tilde{\eta}_{S_{1}}^{2}= & \sum_{K \in \zeta} \alpha_{K}^{2}\left\|f-\frac{\partial u_{h}}{\partial t}+\varepsilon \Delta u_{h}-\mathbf{a} \cdot \nabla u_{h}-b u_{h}\right\|_{L^{2}(K)}^{2}+\sum_{E \in \mathscr{E}_{\text {int }}(\zeta)} \varepsilon^{\frac{3}{2}} \alpha_{E}\left\|\left[\nabla u_{h}\right]\right\|_{L^{2}(E)}^{2} \\
& +\sum_{E \in \mathscr{E}(\zeta)}\left(\frac{\gamma \varepsilon}{h_{E}}+\beta h_{E}+\frac{h_{E}}{\varepsilon}\right)\left\|\left[u_{h}\right]\right\|_{L^{2}(E)}^{2}, \\
\tilde{\eta}_{S_{2}}^{2}= & \sum_{E \in \mathscr{E}(\zeta)} h_{E}\left\|\left[\frac{\partial u_{h}}{\partial t}\right]\right\|_{L^{2}(E)}^{2}, \\
\tilde{\eta}_{S_{3}}^{2}= & \sum_{E \in \mathscr{E}(\zeta)} h_{E}\left\|\left[u_{h}\right]\right\|_{L^{2}(E)}^{2} .
\end{aligned}
$$

THEOREM 5.2 The error $e$ of the semi-discrete dG method (3.5) satisfies the bound

$$
\|e\|_{*} \lesssim \tilde{\eta}
$$

Proof. Choosing $v=e_{c}$ in Lemma 5.1 gives

$$
\left(\frac{\partial e_{c}}{\partial t}, e_{c}\right)+B\left(t ; e_{c}, e_{c}\right)=\left(\frac{\partial u_{h, d}}{\partial t}, e_{c}\right)+B\left(t ; \theta_{c}, e_{c}\right) .
$$

Using (4.2), (4.3), and Young's inequality we arrive to

$$
\frac{d}{d t}\left(\left\|e_{c}\right\|^{2}\right)+\left.||\left|e_{c}\right|\right|^{2} \lesssim\left(||\left|\theta_{c}\right| \|+\left|\theta_{c}\right|_{A}\right)^{2}+\left\|\frac{\partial u_{h, d}}{\partial t}\right\||| e_{c} \| .
$$

Let $T_{0} \in[0, T]$ be such that $E_{c}:=\left\|e_{c}\left(T_{0}\right)\right\|=\left\|e_{c}\right\|_{L^{\infty}\left(0, T ; L^{2}(\Omega)\right)}$ then integrating (5.7) on $[0, T]$ and $\left[0, T_{0}\right]$ gives

$$
\left\|e_{c}\right\|_{*}^{2} \lesssim\left\|e_{c}(0)\right\|^{2}+\int_{0}^{T}\left(||\left|\theta_{c}\right| \|+\left|\theta_{c}\right|_{A}\right)^{2} d t+E_{c} \int_{0}^{T}\left\|\frac{\partial u_{h, d}}{\partial t}\right\| d t .
$$

Using young's inequality on (5.8) gives

$$
\left\|e_{c}\right\|_{*}^{2} \lesssim\left\|e_{c}(0)\right\|^{2}+\int_{0}^{T}\left(\left.\left\||| \theta_{c}\right\||+| \theta_{c}\right|_{A}\right)^{2} d t+\left(\int_{0}^{T}\left\|\frac{\partial u_{h, d}}{\partial t}\right\| d t\right)^{2} .
$$

Going back to (5.7) and using the Poincaré-Friedrichs inequality, Young's inequality and again integrating over $[0, T]$ and $\left[0, T_{0}\right]$ gives:

$$
\left\|e_{c}\right\|_{*}^{2} \lesssim\left\|e_{c}(0)\right\|^{2}+\int_{0}^{T}\left(\left.\left\||| \theta_{c}\right\||+| \theta_{c}\right|_{A}\right)^{2} d t+\alpha_{T}^{2} \int_{0}^{T}\left\|\frac{\partial u_{h, d}}{\partial t}\right\|^{2} d t .
$$

Combining (5.9) and (5.10) and using the triangle inequality yields

$$
\begin{aligned}
\|e\|_{*}^{2} & \lesssim\|e(0)\|^{2}+\min \left\{\left(\int_{0}^{T}\left\|\frac{\partial u_{h, d}}{\partial t}\right\| d t\right)^{2}, \alpha_{T}^{2} \int_{0}^{T}\left\|\frac{\partial u_{h, d}}{\partial t}\right\|^{2} d t\right\} \\
& +\int_{0}^{T}\left(\|\| \theta \|\left.|+| \theta\right|_{A}\right)^{2} d t+\left\|u_{h, d}\right\|_{*}^{2} .
\end{aligned}
$$

The proof then follows directly from Theorem 4.1 and (5.3). 
8 of 19

\section{An a posteriori bound for the fully-discrete method}

We now continue by applying to the fully-discrete setting the general framework presented in the previous section.

Definition 6.1 We define $A^{k} \in V_{h}^{k}$ to be the unique solution of the elliptic problem

$$
B\left(t^{k} ; u_{h}^{k}, v_{h}^{k}\right)+K_{h}\left(u_{h}^{k}, v_{h}^{k}\right)=\left(A^{k}, v_{h}^{k}\right) \quad \forall v_{h}^{k} \in V_{h}^{k}
$$

REMARK 6.1 For $k \geqslant 1$ we obtain from the numerical method that $A^{k+1}=I_{h}^{k+1} f^{k+1}-\frac{u_{h}^{k+1}-I_{h}^{k+1} u_{h}^{k}}{\tau_{k+1}}$ where $I_{h}^{k+1}$ is the $L^{2}$-projection operator onto $V_{h}^{k+1}$.

DEFINITION 6.2 We define the elliptic reconstruction $w^{k} \in H_{0}^{1}(\Omega)$ to be the unique solution of the elliptic problem

$$
B\left(t^{k} ; w^{k}, v\right)=\left(A^{k}, v\right) \quad \forall v \in H_{0}^{1}(\Omega)
$$

At each time step $k$, we decompose the $\mathrm{dG}$ solution $u_{h}^{k}$ into a conforming part $u_{h, c}^{k} \in H_{0}^{1}(\Omega) \cap V_{h}^{k}$ and a non-conforming part $u_{h, d}^{k} \in V_{h}^{k}$ such that $u_{h}^{k}=u_{h, c}^{k}+u_{h, d}^{k}$. Given $t \in\left(t^{k}, t^{k+1}\right]$, we (re)define $u_{h}(t)$ to be the linear interpolant with respect to $t$ of the values $u_{h}^{k}$ and $u_{h}^{k+1}$, viz.,

$$
u_{h}(t):=l_{k}(t) u_{h}^{k}+l_{k+1}(t) u_{h}^{k+1}
$$

where $\left\{l_{k}, l_{k+1}\right\}$ denotes the standard linear Lagrange interpolation basis defined on the interval $\left[t^{k}, t^{k+1}\right]$. We define $u_{h, c}(t)$ and $u_{h, d}(t)$ analogously. We can then decompose the error $e=u-u_{h}=e_{c}-u_{h, d}$ where $e_{c}=u-u_{h, c}$. It will also be useful to define $\theta^{k}=w^{k}-u_{h}^{k}$.

LEMMA 6.1 Given $t \in\left(t^{k}, t^{k+1}\right]$ we have

$$
\left(\frac{\partial e}{\partial t}, v\right)+B(t ; e, v)=\left(f-f^{k+1}, v\right)+\left(f^{k+1}-\frac{\partial u_{h}}{\partial t}, v\right)-B\left(t ; u_{h}, v\right) \quad \forall v \in H_{0}^{1}(\Omega) .
$$

Proof. This follows from Definition 6.2 and (3.8) and by rearranging the resulting equation. Before proving the a posteriori bounds for the fully-discrete method, we introduce the error estimators. We begin by defining the spatial estimator, $\eta_{S}$, by

$$
\begin{aligned}
\eta_{S}^{2} & =\|e(0)\|^{2}+\frac{1}{3} \sum_{j=0}^{n-1} \tau_{j+1}\left(\eta_{S_{1}, j}^{2}+\eta_{S_{1}, j+1}^{2}\right)+\sum_{j=0}^{n-1} \tau_{j+1} \eta_{S_{2}, j+1}^{2}+\max _{0 \leqslant j \leqslant n} \eta_{S_{3}, j}^{2} \\
& +\min \left\{\left(\sum_{j=0}^{n-1} \tau_{j+1} \eta_{S_{4}, j+1}\right)^{2}, \alpha_{T}^{2} \sum_{j=0}^{n-1} \tau_{j+1} \eta_{S_{4}, j+1}^{2}\right\},
\end{aligned}
$$


where

$$
\begin{aligned}
\eta_{S_{1}, j}^{2} & =\sum_{K \in \zeta^{j}} \alpha_{K}^{2}\left\|A^{j}+\varepsilon \Delta u_{h}^{j}-\mathbf{a}^{j} \cdot \nabla u_{h}^{j}-b^{j} u_{h}^{j}\right\|_{L^{2}(K)}^{2}+\sum_{E \in \mathscr{E}^{\text {int }}\left(\zeta^{j}\right)} \varepsilon^{\frac{3}{2}} \alpha_{E}\left\|\left[\nabla u_{h}^{j}\right]\right\|_{L^{2}(E)}^{2} \\
& +\sum_{E \in \mathscr{E}\left(\zeta^{j}\right)}\left(\frac{\gamma \varepsilon}{h_{E}}+\beta h_{E}+\frac{h_{E}}{\varepsilon}\right)\left\|\left[u_{h}^{j}\right]\right\|_{L^{2}(E)}^{2}, \\
\eta_{S_{2}, j+1}^{2} & =\sum_{K \in \zeta^{j} \cup \zeta^{j+1}} \alpha_{K}^{2}\left\|f^{j+1}-I_{h}^{j+1} f^{j+1}+\frac{u_{h}^{j}-I_{h}^{j+1} u_{h}^{j}}{\tau_{j+1}}\right\|_{L^{2}(K)}^{2} \\
\eta_{S_{3}, j}^{2}= & \sum_{E \in \mathscr{E}\left(\zeta^{j}\right)} h_{E}\left\|\left[u_{h}^{j}\right]\right\| \|_{L^{2}(E)}^{2}, \\
\eta_{S_{4}, j+1}^{2}= & \left.\sum_{E \in \mathscr{E}\left(\zeta^{j} \cup \zeta^{j+1}\right)} h_{E}\left\|\left[\frac{u_{h}^{j+1}-u_{h}^{j}}{\tau_{j+1}}\right]\right\|\right|_{L^{2}(E)} ^{2} .
\end{aligned}
$$

The time (or temporal) estimator, $\eta_{T}$ is given by

$$
\eta_{T}^{2}=\sum_{j=0}^{n-1} \int_{t^{j}}^{t^{j+1}} \eta_{T_{1}, j+1}^{2} d t+\min \left\{\left(\sum_{j=0}^{n-1} \int_{t^{j}}^{t^{j+1}} \eta_{T_{2}, j+1} d t\right)^{2}, \alpha_{T}^{2} \sum_{j=0}^{n-1} \int_{t^{j}}^{t^{j+1}} \eta_{T_{2}, j+1}^{2} d t\right\} .
$$

where

$$
\begin{aligned}
\eta_{T_{1}, j+1}^{2} & =\sum_{K \in \zeta^{j} \cup \zeta^{j+1}} \frac{1}{\varepsilon}\left\|l_{j+1}\left(\mathbf{a}^{j+1}-\mathbf{a}\right) u_{h}^{j+1}+l_{j}\left(\mathbf{a}^{j}-\mathbf{a}\right) u_{h}^{j}\right\|_{L^{2}(K)}^{2}, \\
\eta_{T_{2}, j+1}^{2}= & \sum_{K \in \zeta^{j} \zeta^{j+1}} \| f-f^{j+1}+l_{j}\left(A^{j+1}-A^{j}\right)+l_{j+1}\left(b^{j+1}-b-\nabla \cdot \mathbf{a}^{j+1}+\nabla \cdot \mathbf{a}\right) u_{h}^{j+1} \\
& +l_{j}\left(b^{j}-b-\nabla \cdot \mathbf{a}^{j}+\nabla \cdot \mathbf{a}\right) u_{h}^{j} \|_{L^{2}(K)}^{2} .
\end{aligned}
$$

THEOREM 6.3 The error $e$ of the fully-discrete method satisfies the bound

$$
\|e\|_{*} \lesssim \sqrt{\eta_{S}^{2}+\eta_{T}^{2}}
$$

Proof. From Lemma 6.1 and Definition 6.2 we have

$$
\begin{aligned}
\left(\frac{\partial e}{\partial t}, v\right)+B(t ; e, v) & =\left(f-f^{k+1}, v\right)+\left(f^{k+1}-\frac{\partial u_{h}}{\partial t}-A^{k+1}, v\right)+B\left(t^{k+1} ; \theta^{k+1}, v\right) \\
& +B\left(t^{k+1} ; u_{h}^{k+1}, v\right)-B\left(t ; u_{h}, v\right)
\end{aligned}
$$

which upon straightforward manipulation and using Remark 6.1 gives

$$
\begin{aligned}
\left(\frac{\partial e}{\partial t}, v\right)+B(t ; e, v) & =l_{k+1} B\left(t^{k+1} ; \theta^{k+1}, v\right)+l_{k} B\left(t^{k} ; \theta^{k}, v\right)+\left(f-f^{k+1}+l_{k}\left(A^{k+1}-A^{k}\right), v\right) \\
& +l_{k+1} B\left(t^{k+1} ; u_{h}^{k+1}, v\right)+l_{k} B\left(t^{k} ; u_{h}^{k}, v\right)-B\left(t ; u_{h}, v\right)+\left(f^{k+1}-\frac{\partial u_{h}}{\partial t}-A^{k+1}, v\right) .
\end{aligned}
$$

The first 2 terms give rise to the space estimator via elliptic reconstruction, the final term can be rewritten using Remark 6.1, properties and bounds of the $L^{2}$-projection and the Cauchy-Schwarz inequality:

$$
\left(f^{k+1}-\frac{\partial u_{h}}{\partial t}-A^{k+1}, v\right)=\left(f^{k+1}-\frac{\partial u_{h}}{\partial t}-A^{k+1}, v-I_{h}^{k+1} v\right) \lesssim \eta_{S_{2}, k+1}\|\mid v\| \|,
$$


10 of 19

By combining terms, using the definition of the bilinear form $B$ and using the Cauchy-Schwarz inequality; the remaining four terms give rise to the time estimator:

$$
\begin{aligned}
& \left(f-f^{k+1}+l_{k}\left(A^{k+1}-A^{k}\right), v\right)+l_{k+1} B\left(t^{k+1} ; u_{h}^{k+1}, v\right)+l_{k} B\left(t^{k} ; u_{h}^{k}, v\right) \\
& -B\left(t ; u_{h}, v\right) \lesssim \eta_{T_{1}, k+1}\|v\|\left\|+\eta_{T_{2}, k+1}\right\| v \| .
\end{aligned}
$$

Setting $v=e_{c}$ and using (4.2), (4.3), the Cauchy-Schwarz inequality and Young's inequality yields

$$
\begin{aligned}
\frac{d}{d t}\left(\left\|e_{c}\right\|^{2}\right)+\left|\left\|e_{c}\right\|\right|^{2} \lesssim & \left\|\frac{\partial u_{h, d}}{\partial t}\right\|\left\|e_{c}\right\|+l_{k+1}^{2}\left(\left\|\left.|| \theta^{k+1}|\|+| \theta^{k+1}\right|_{A}\right)^{2}+l_{k}^{2}\left(\left|\left\|\left.\theta^{k}|\|+| \theta^{k}\right|_{A}\right)^{2}\right.\right.\right. \\
& +\left.\left\||| u_{h, d}\right\|\right|^{2}+\left|u_{h, d}\right|_{A}^{2}+\eta_{T_{2}, k+1}\left\|e_{c}\right\|+\eta_{T_{1}, k+1}^{2}+\eta_{S_{2}, k+1}^{2}
\end{aligned}
$$

The proof then follows from Theorem 4.1 and (5.3) and by employing a bounding strategy identical to that used in Theorem 5.2.

REMARK 6.2 The spatial estimator is expected to be robust, in the sense that the effectivity indices (i.e., the ratio between the true errors and the value of the a posteriori estimators) are insensitive with respect to $\varepsilon$, once the spatial mesh is sufficiently resolved as the predominant terms are the same as in the elliptic case. For pre-asymptotic case, one would need to work in stronger norms to achieve theoretical robustness. We note, however, that in all the numerical experiments below, the adaptive algorithm, implementing the estimators presented here, was able to arrive to optimally convergent spacetime mesh modifications. The temporal error estimator is also expected to be robust with respect to $\varepsilon$ in the asymptotic regime as the temporal data approximation error terms are all order two in time and the only order one temporal term is a difference of derivatives (from Remark 6.1) which is anticipated to be independent of $\varepsilon$ in the asymptotic regime.

REMARK 6.3 The use of elliptic reconstruction is not essential for the proof of Theorem 5.2 and Theorem 6.3. It is possible to derive the residual based a posteriori bounds directly albeit at the cost of a lengthier calculation. The advantage of using the elliptic reconstruction in the proof lies in the fact that it can be easily modified to include non-residual based spatial a posteriori estimators. This, in turn, may offer improvements in robustness with respect to the Péclet number (cf. Sangalli (2008)).

\section{An adaptive algorithm}

The a posteriori bounds presented above will be used to drive a space-time adaptive algorithm. A number of adaptive algorithms for parabolic problems have been proposed in the literature; see e.g. Chen \& Feng (2004); Schmidt \& Siebert (2005) and the references therein.

Here, we propose a variant of the adaptive algorithms from Chen \& Feng (2004); Schmidt \& Siebert (2005) which appears to perform well for our discretisation. The pseudocode is given in Algorithm 1. The algorithm is based on using different parts of the a posteriori estimator from Theorem 6.3 to drive space-time adaptivity. In particular, in contrast with Chen \& Feng (2004); Schmidt \& Siebert (2005) the adaptive algorithm utilises the spatial estimator for both refinement and coarsening in space.

Both mesh refinement and coarsening are driven by the term $\eta_{S_{1}, j+1}$. The size of the elemental contributions to $\eta_{S_{1}, j+1}$ determines whether the elements are to be refined, coarsened or neither depending on two spatial thresholds stol ${ }^{+}$and stol ${ }^{-}$. The nature of the time estimator, $\eta_{T}$, makes it difficult to use as a temporal refinement indicator so, to this end we define $\hat{\eta}_{T, j+1}$ given by

$$
\hat{\eta}_{T, j+1}^{2}=\int_{t^{j}}^{t^{j+1}} \eta_{T_{1}, j+1}^{2} d t+\min \left\{\alpha_{T}, T\right\} \int_{t^{j}}^{t^{j+1}} \eta_{T_{2}, j+1}^{2} d t
$$




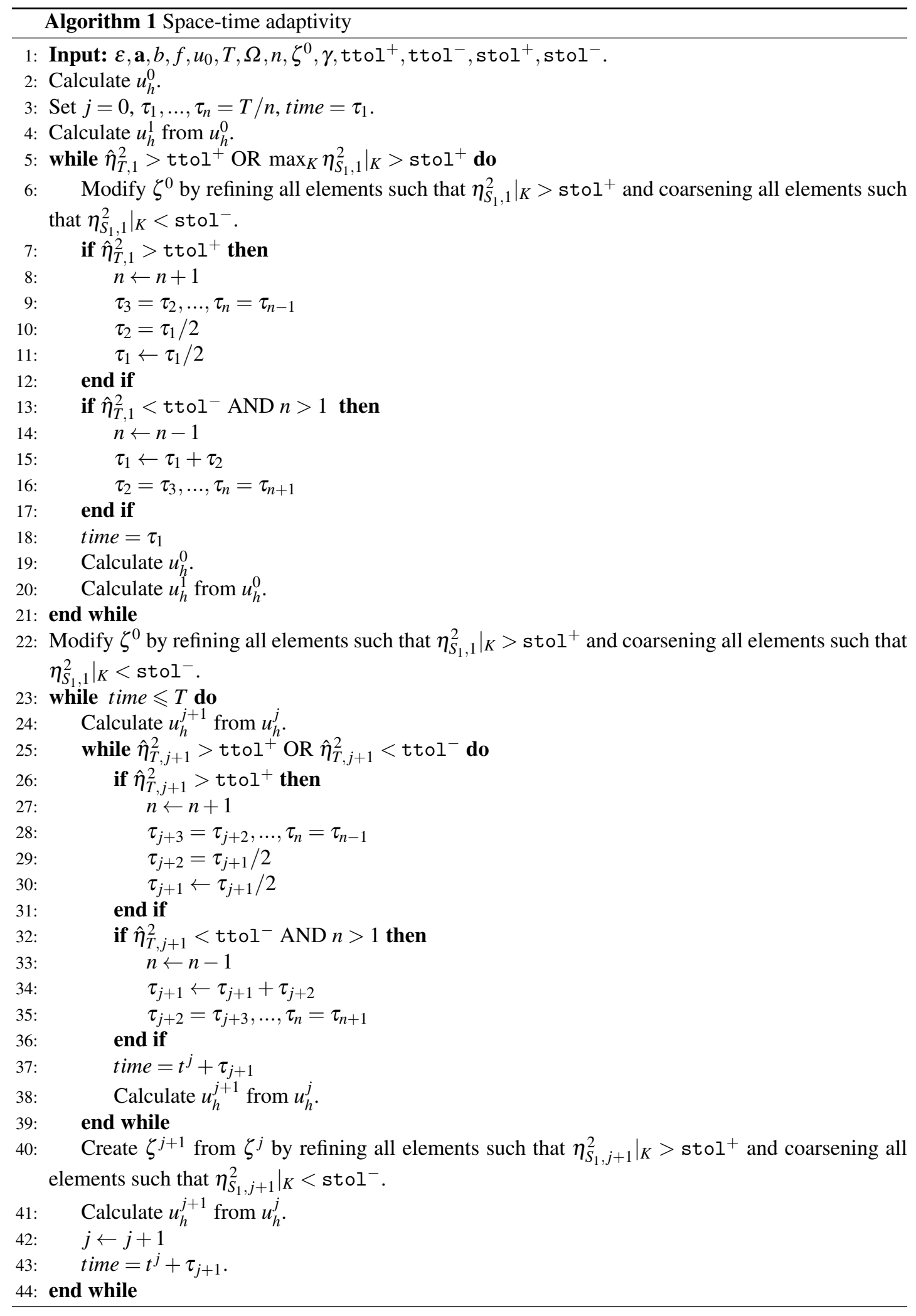


The sum of all terms $\hat{\eta}_{T, j+1}^{2}$ bound $\eta_{T}^{2}$ and hence can be used to drive temporal refinement and coarsening subject to two temporal thresholds ttol ${ }^{+}$and $\mathrm{ttol}^{-}$on each time interval.

REMARK 7.1 Mesh modification must be done very carefully to ensure the solution does not degrade. The spatial refinement threshold stol ${ }^{+}$needs to be chosen sufficiently small in comparison to the temporal refinement threshold ttol $1^{+}$; this is to ensure that the terms $\eta_{3, j+1}^{2}$ and $\eta_{4, j+1}^{2}$ are sufficiently small. Secondly, the spatial coarsening threshold stol ${ }^{-}$and the temporal coarsening threshold ttol ${ }^{-}$ need to be chosen sufficiently small in comparison to the spatial refinement threshold stol ${ }^{+}$and the temporal refinement threshold ttol ${ }^{+}$to avoid unnecessary refine and then coarsen loops.

REMARK 7.2 We stress that even when ttol- is set to be equal to 0 , the algorithm does not necessarily produce monotonically decreasing time step distribution from 0 to $T$. Indeed, the algorithm starts with an initial equispaced subdivision of $[0, T]$ into $n$ time intervals, which is then, possibly, locally bisected based on ttol ${ }^{+}$. For instance, if the solution reaches a smoothly varying steady state, the algorithm will retain the original (coarse) time step of size $T / n$ at the final stages of the computation.

\section{Numerical experiments}

We shall investigate numerically the presented a posteriori bounds and the performance of the adaptive algorithm through an implementation based on the deal. I I finite element library (Bangerth et al. (2007)). All the numerical experiments have been performed using the high performance computing facility ALICE at the University of Leicester.

We denote by $\lambda_{k}$ the total number of degrees of freedom on the union mesh $\zeta^{k} \cup \zeta^{k+1}$. Hence, the weighted degrees of freedom of the problem is given by

$$
\text { Weighted Average DoFs }:=\frac{1}{T} \sum_{j=0}^{n-1} \tau_{j+1} \lambda_{j} \text {. }
$$

In all examples presented below, unless otherwise stated, $p=2, \gamma=10$, stol ${ }^{-}=0.001 *$ stol $^{+}$, ttol ${ }^{-}=0$ and $\zeta^{0}$ is a $4 \times 4$ uniform quadrilateral mesh. Finally, unmarked lines in convergence plots represent the theoretically expected optimal rate of convergence for reference.

\subsection{Example 1}

Let $\Omega=(0,1)^{2}, \mathbf{a}=(1,1)^{T}, b=0, u_{0}=0, T=10$ and select the function $f$ so that the exact solution to problem (2.5) is given by

$$
u(x, y, t)=\left(1-e^{-t}\right)\left(\frac{e^{(x-1) / \varepsilon}-1}{e^{-1 / \varepsilon}-1}+x-1\right)\left(\frac{e^{(y-1) / \varepsilon}-1}{e^{-1 / \varepsilon}-1}+y-1\right) .
$$

The solution exhibits boundary layers at the outflow boundary of the domain of width $\mathscr{O}(\varepsilon)$ as well as a temporal boundary layer.

We begin by fixing a temporal threshold that produces enough time steps so that the temporal contribution to the error is very small in comparison to the spatial contribution. The spatial threshold is then gradually reduced to observe the spatial effectivity indices for this problem which are given in Figure 1. Optimal rates of convergence are observed with respect to the weighted average degrees of freedom for both the estimator and the error but are omitted in this example. As shown, the effectivity indices 

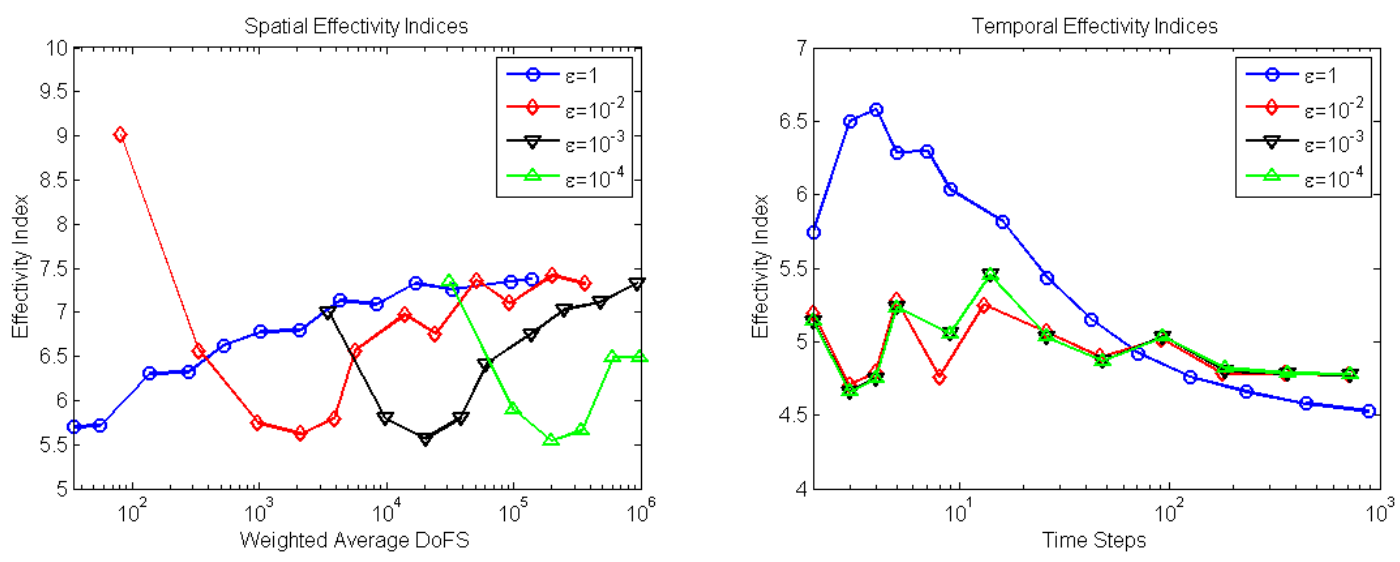

FIG. 1. Example 1: Spatial and temporal effectivity indices.

are bounded asymptotically and remain between 5 and 10 for the different values of $\varepsilon$; these are directly comparable to those observed in Schötzau \& Zhu (2009) for the stationary problem.

In order to study the temporal effectivity indices for this problem any boundary layers must be fully resolved so that the spatial error is dominated by the temporal one. To this end, we use a high polynomial degree with sufficiently large penalty parameter $\gamma$ and use specially constructed anisotropic meshes in order to ensure that the spatial error is sufficiently small. The temporal threshold is then reduced to observe the temporal effectivity indices of the problem which are given in Figure 1. Optimal order is observed with respect to both the estimator and the error and the effectivity indices remain bounded between 4 and 7 for all values of $\varepsilon$.

The presence of a temporal boundary layer in the solution motivates a comparison between adaptive and uniform time-stepping. To this end, a sufficiently small spatial threshold is chosen so that the spatial contribution to the error is small and then the temporal threshold is decreased and the results are compared to just using uniform time-stepping. The results given in Figure 2 show that the temporal strategy of the adaptive algorithm minimizes the temporal portion of the error better than just using uniform time stepping.

\subsection{Example 2}

We set $\Omega=(-1,1)^{2}, \mathbf{a}=(1,1)^{T}, b=1, f=\sin (5 t) x y, u_{0}=0$ and $T=2 \pi$. The solution exhibits layers of width $\mathscr{O}(\varepsilon)$ in the proximity of the outflow boundary and is oscillatory in time. The sharpness of the boundary layers depend on time, thus making this a good test of the ability of the algorithm to add and remove degrees of freedom. As in Example 1, we begin by fixing a temporal threshold while decreasing the spatial threshold to observe the rates of convergence for the space estimator. We then set a spatial threshold small enough to resolve any boundary layers, while reducing the temporal threshold to observe the rates of the time estimator. The results are displayed in Figure 3. Optimal rates of convergence are observed for both the space and the time estimators.

To assess the mesh change driven by the adaptive algorithm we also plot the individual degrees of freedom on each mesh against time for a given spatial and temporal threshold. The results are given in Figure 4. We observe that the adaptive algorithm is adding and removing degrees of freedom at a rate 
14 of 19


FIG. 2. Example 1: Temporal error comparison under adaptive and uniform time-stepping for $\varepsilon=1$ and $\varepsilon=10^{-2}$.
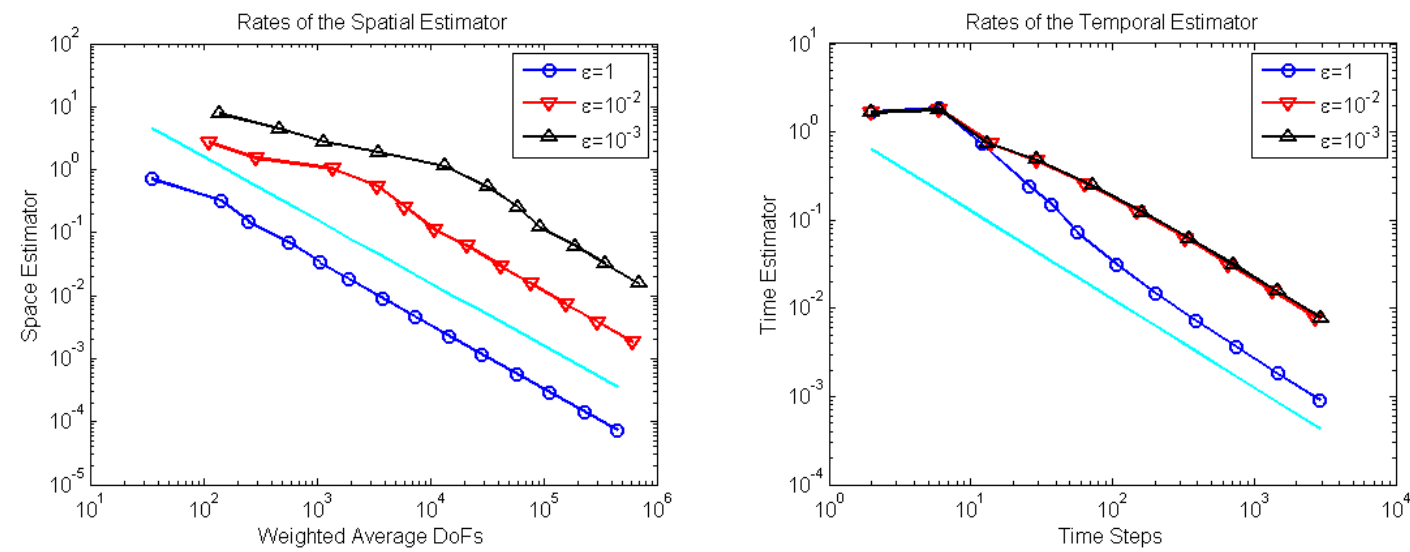

FIG. 3. Example 2: Spatial and temporal rates.

that is in accordance with the oscillating nature of the solution driven by the sinusoidal forcing $f$.

\subsection{Example 3}

Let $\Omega=(-2,2)^{2}, T=2 \pi, \mathbf{a}=(y,-x)^{T}, b=0, f=0$ and $u_{0}=e^{-64(x-0.5)^{2}} e^{-64 y^{2}}$. The PDE convects the initial two dimensional Gaussian profile along the circular wind while diffusing it at a rate depending upon $\varepsilon$. In particular, providing the error at the boundary is sufficiently small, the exact solution to problem (2.5) is given by

$$
u(x, y, t)=\frac{1}{1+256 \varepsilon t} \exp \left(-\frac{64(x-0.5 \cos (t))^{2}}{1+256 \varepsilon t}\right) \exp \left(-\frac{64(y+0.5 \sin (t))^{2}}{1+256 \varepsilon t}\right) .
$$

To observe the temporal effectivity indices and temporal rates of the problem we first fix a spatial threshold so that the spatial contribution to the error is small and then reduce the temporal threshold; 

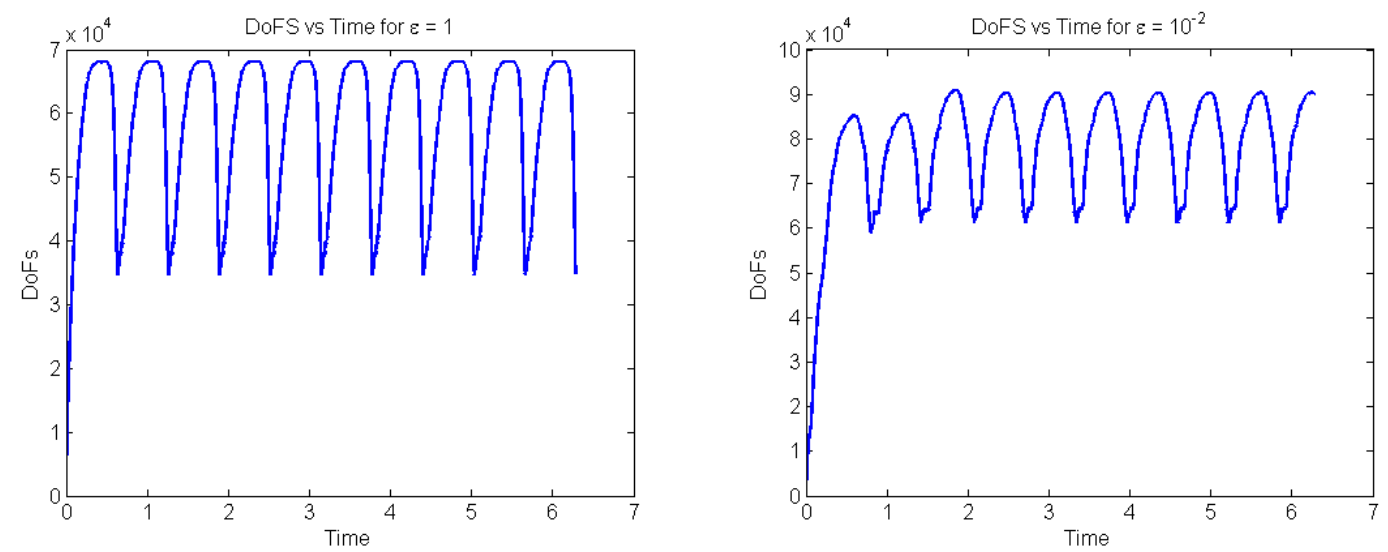

FIG. 4. Example 2: DoFS vs Time for $\varepsilon=1$ and $\varepsilon=10^{-2}$.
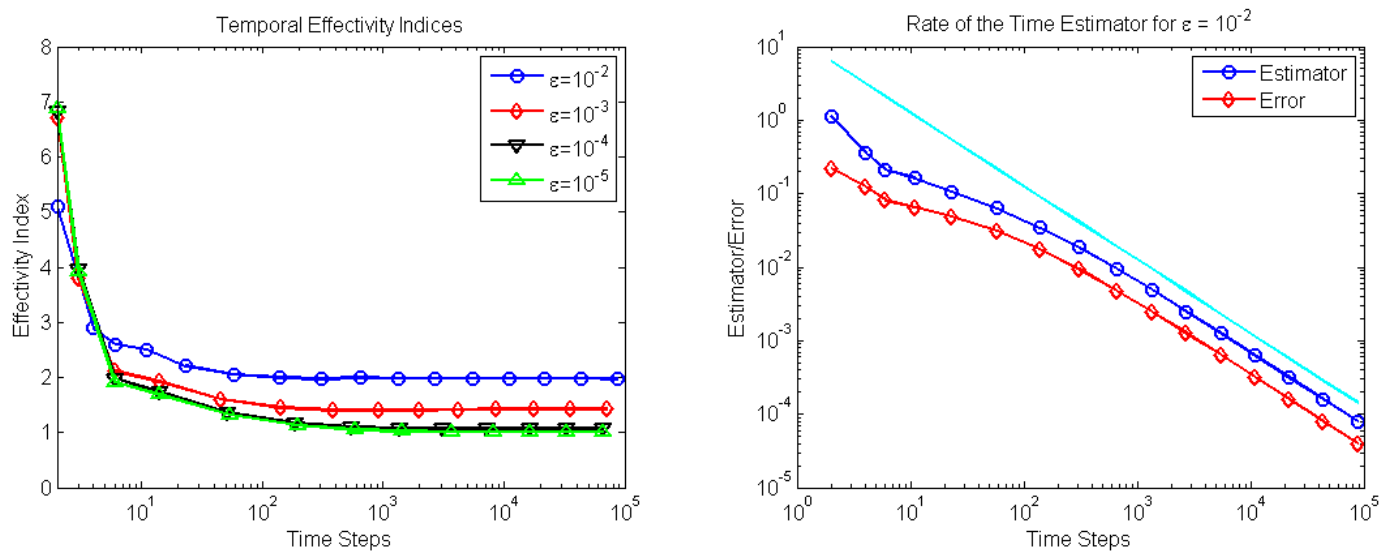

FIG. 5. Example 3: Temporal effectivity indices and the rate of the time estimator for $\varepsilon=10^{-2}$.

the results given in Figure 5 show that the temporal effectivity indices are bounded and remain between 1 and 8 for all values of $\varepsilon$ and that the optimal rate of convergence is achieved by both the error and the estimator. Some meshes at various time steps produced by the algorithm for $\varepsilon=10^{-5}$ are shown in Figure 6 and show that the adaptive algorithm is adding and removing degrees of freedom efficiently.

\subsection{Example 4}

Let $\Omega=(0,1)^{2}, \mathbf{a}=(\sin (t), \cos (t))^{T}, b=0, f=1, u_{0}=0$ and $T=2 \pi$. The nature of the solution is rather uniform in time but has a boundary layer of width $\mathscr{O}(\varepsilon)$ whose location depends on time. Therefore, this example is well suited to test the ability of the algorithm to adapt the grid to this moving boundary layer as time evolves. See Figure 7 where grids at various times for $\varepsilon=10^{-2}$ are shown.

As in previous examples, we fix a temporal threshold and then reduce the spatial threshold to observe the rates of the space estimator. Again, we also fix a spatial threshold small enough to ensure that all 
16 of 19
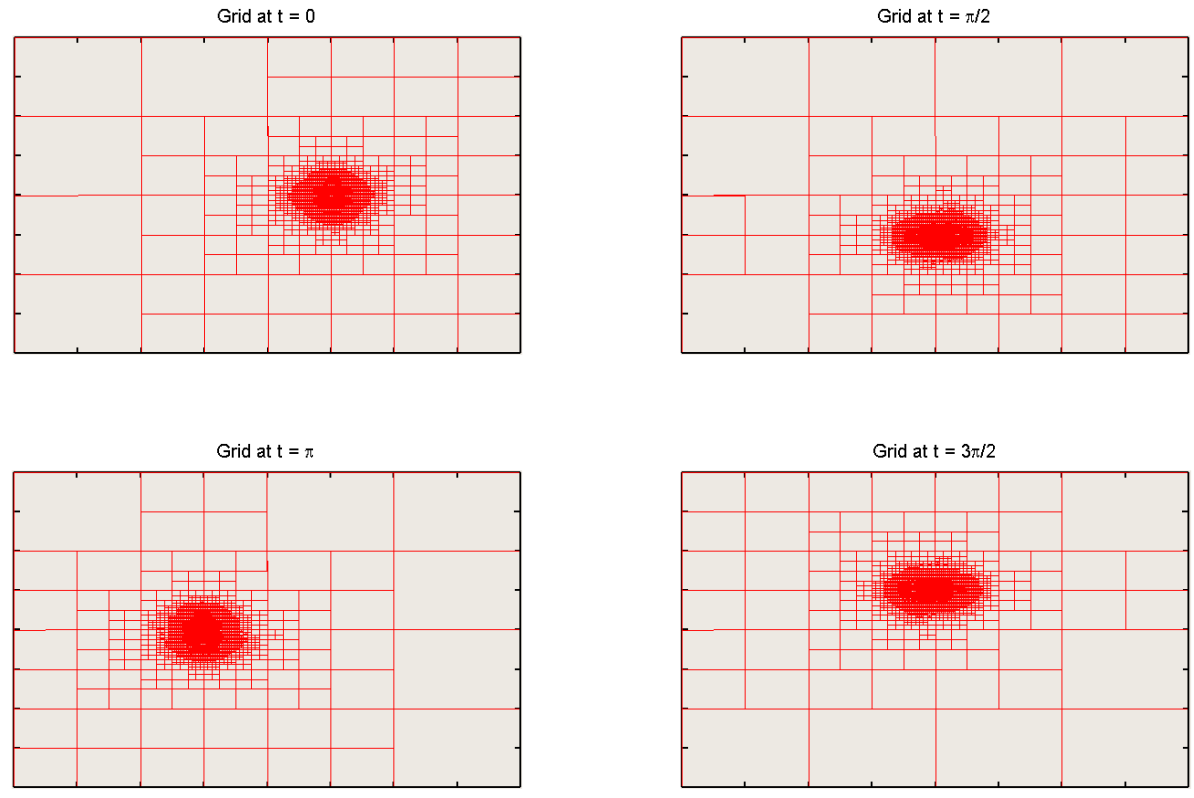

FIG. 6. Example 3: Grid snapshots.
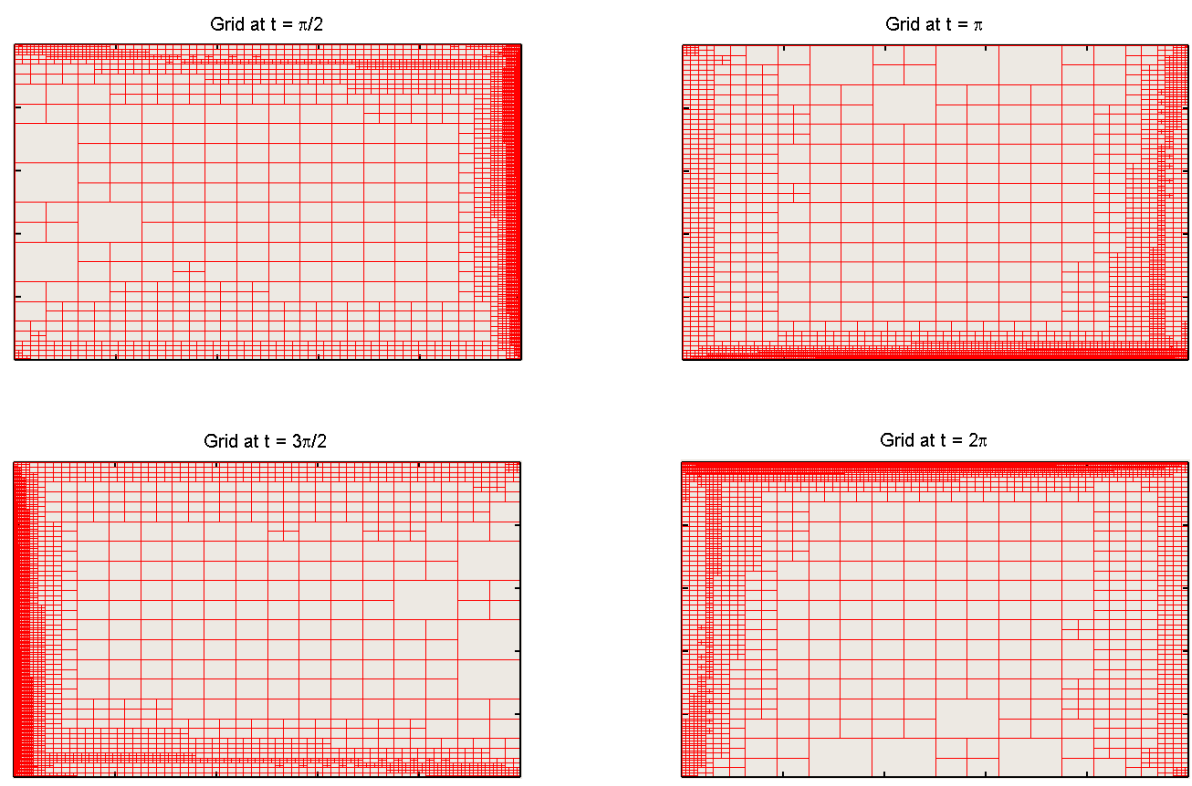

FIG. 7. Example 4: Grid snapshots. 

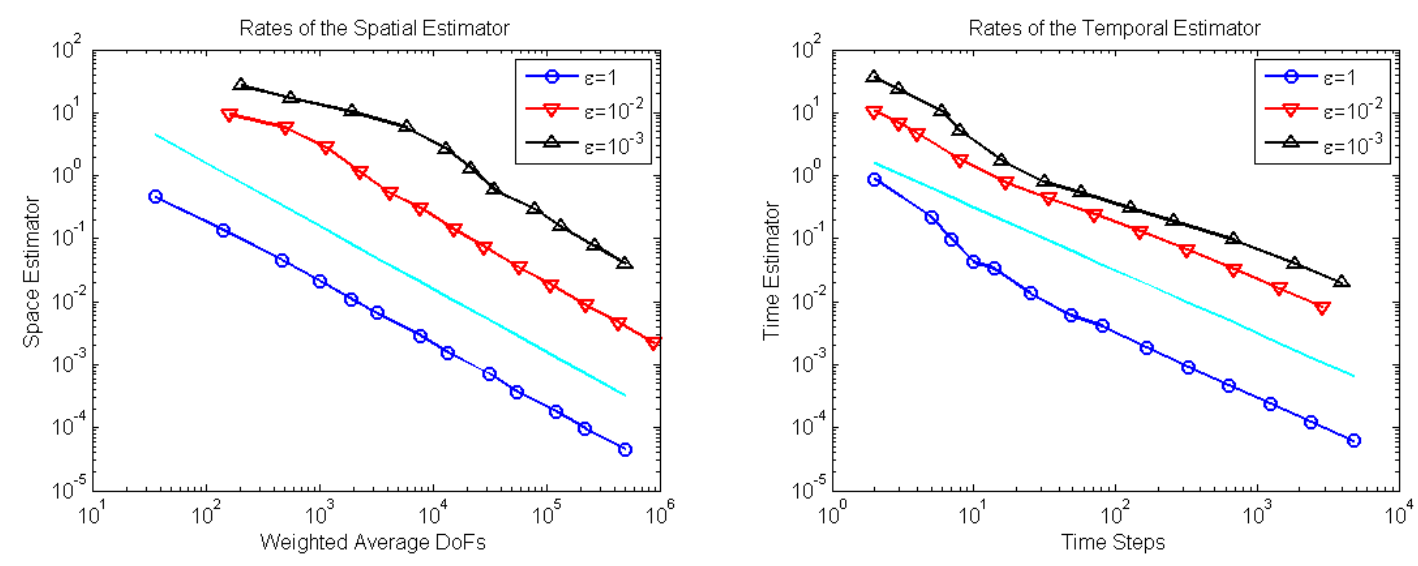

FIG. 8. Example 4: Spatial and temporal rates.

boundary layers are sufficiently resolved and then observe the rates of the time estimator. These results are given in Figure 8. Optimal spatial and temporal rates of convergence are observed and the grids produced for $\varepsilon=10^{-2}$ clearly show that the adaptive algorithm is picking up the boundary layers as they move around the domain and that unneeded degrees of freedom are not retained.

\section{Conclusions}

An a posteriori error estimator for the discontinuous Galerkin spatial discretisation of a non-stationary linear convection-diffusion equation is presented. The derivation of the estimator is based on reconstruction techniques to make use of robust a posteriori estimators for elliptic problems already developed in the literature. Our numerical examples clearly indicate that the error estimator is practical and the respective space-time adaptive algorithm works well for the studied problems. The spatial effecitivity indices are in an identical range to those observed in (Schötzau \& Zhu (2009)) and the temporal effectivity indices are substantially smaller than the ones seen in (Georgoulis et al. (2011)) for the heat problem.

\section{REFERENCES}

Ainsworth, M. \& Oden, J. T. (2000) A posteriori error estimation in finite element analysis. Pure and Applied Mathematics (New York). New York: Wiley-Interscience [John Wiley \& Sons], pp. xx+240.

Araya, R., Behrens, E. \& Rodríguez, R. (2005a) An adaptive stabilized finite element scheme for the advection-reaction-diffusion equation. Appl. Numer. Math., 54, 491-503.

Araya, R., PozA, A. H. \& Stephan, E. P. (2005b) A hierarchical a posteriori error estimate for an advectiondiffusion-reaction problem. Math. Models Methods Appl. Sci., 15, 1119-1139.

Bangerth, W., Hartmann, R. \& Kanschat, G. (2007) deal.II-a general-purpose object-oriented finite element library. ACM Trans. Math. Software, 33, Art. 24, 27.

Becker, R., HANsbo, P. \& LARson, M. G. (2003) Energy norm a posteriori error estimation for discontinuous Galerkin methods. Comput. Methods Appl. Mech. Engrg., 192, 723-733.

Berrone, S. \& CANuto, C. (2004) Multilevel a posteriori error analysis for reaction-convection-diffusion problems. Appl. Numer. Math., 50, 371-394.

Chen, Z. \& Feng, J. (2004) An adaptive finite element algorithm with reliable and efficient error control for 
18 of 19

linear parabolic problems. Math. Comp., 73, 1167-1193 (electronic).

Dolejší, V., Ern, A. \& Vohralík, M. (2013) A Framework for Robust A Posteriori Error Control in Unsteady Nonlinear Advection-Diffusion Problems. SIAM J. Numer. Anal., 51, 773-793.

Dupont, T. (1982) Mesh modification for evolution equations. Math. Comp., 39, 85-107.

Ern, A., Stephansen, A. F. \& Vohralík, M. (2010) Guaranteed and robust discontinuous Galerkin a posteriori error estimates for convection-diffusion-reaction problems. J. Comput. Appl. Math., 234, 114-130.

ERN, A. \& Proft, J. (2005) A posteriori discontinuous Galerkin error estimates for transient convection-diffusion equations. Appl. Math. Lett., 18, 833-841.

ERn, A. \& Vohralí́, M. (2010) A posteriori error estimation based on potential and flux reconstruction for the heat equation. SIAM J. Numer. Anal., 48, 198-223.

Georgoulis, E. H., HAll, E. \& Houston, P. (2008) Discontinuous Galerkin methods for advection-diffusionreaction problems on anisotropically refined meshes. SIAM J. Sci. Comput., 30, 246-271.

Georgoulis, E. H., LakKis, O. \& Virtanen, J. M. (2011) A posteriori error control for discontinuous Galerkin methods for parabolic problems. SIAM J. Numer. Anal., 49, 427-458.

Houston, P., SchÖTZAU, D. \& WiHLER, T. P. (2007) Energy norm a posteriori error estimation of $h p$-adaptive discontinuous Galerkin methods for elliptic problems. Math. Models Methods Appl. Sci., 17, 33-62.

Houston, P. \& SüLI, E. (2001) Adaptive Lagrange-Galerkin methods for unsteady convection-diffusion problems. Math. Comp., 70, 77-106.

Karakashian, O. A. \& PASCAL, F. (2003) A posteriori error estimates for a discontinuous Galerkin approximation of second-order elliptic problems. SIAM J. Numer. Anal., 41, 2374-2399 (electronic).

KUnERT, G. (2003) A posteriori error estimation for convection dominated problems on anisotropic meshes. Math. Methods Appl. Sci., 26, 589-617.

Lozinski, A., Picasso, M. \& Prachittham, V. (2009) An anisotropic error estimator for the Crank-Nicolson method: application to a parabolic problem. SIAM J. Sci. Comput., 31, 2757-2783.

MAKRIDAKIS, C. \& NochetTO, R. H. (2003) Elliptic reconstruction and a posteriori error estimates for parabolic problems. SIAM J. Numer. Anal., 41, 1585-1594.

Roos, H.-G., STynes, M. \& ToBiska, L. (2008) Robust numerical methods for singularly perturbed differential equations. Springer Series in Computational Mathematics, vol. 24, second edn. Berlin: Springer-Verlag, pp. xiv+604. Convection-diffusion-reaction and flow problems.

SAngalli, G. (2008) Robust a-posteriori estimator for advection-diffusion-reaction problems. Math. Comp., 77, 41-70 (electronic).

Schmidt, A. \& Siebert, K. G. (2005) Design of adaptive finite element software. Lecture Notes in Computational Science and Engineering, vol. 42. Berlin: Springer-Verlag, pp. xii+315. The finite element toolbox ALBERTA, With 1 CD-ROM (Unix/Linux).

SchÖтZAU, D. \& ZHU, L. (2009) A robust a-posteriori error estimator for discontinuous Galerkin methods for convection-diffusion equations. Appl. Numer. Math., 59, 2236-2255.

SCHÖTZAU, D. \& ZHU, L. (2011) A robust a posteriori error estimate for $h p$-adaptive DG methods for convectiondiffusion equations. IMA J. Numer. Anal., 31, 971-1005.

ŠEbestovÁ, I. (2012) Two-sided a posteriori error estimates for the DGMs for the heat equation. In A. Cangiani, R.L. Davidchack, E.H. Georgoulis, A. Gorban, J. Levesley, M. Tretyakov (eds.), Numerical Mathematics and Advanced Applications, Proceedings of the ENUMATH 2011 Conference, Leicester. Springer.

Sun, S. \& WheEler, M. F. (2006) A posteriori error estimation and dynamic adaptivity for symmetric discontinuous Galerkin approximations of reactive transport problems. Comput. Methods Appl. Mech. Engrg., 195, 632-652.

VERfürth, R. (1996) A Review of A Posteriori Error Estimation and Adaptive Mesh-Refinement Techniques. Wiley-Teubner, Chichester-Stuttgart, pp. vi+128.

Verfürth, R. (1998) A posteriori error estimators for convection-diffusion equations. Numer. Math., 80, 641663. 
VERFÜRTH, R. (2005a) Robust a posteriori error estimates for nonstationary convection-diffusion equations. SIAM J. Numer. Anal., 43, 1783-1802 (electronic).

VERFÜRTH, R. (2005b) Robust a posteriori error estimates for stationary convection-diffusion equations. SIAM J. Numer. Anal., 43, 1766-1782 (electronic). 